\title{
Histone Deacetylases Take Center Stage on Regulation of Podocyte Function
}

\author{
Min Liu Zhe Qiao Yang Zhang Ping Zhan Fan Yi
}

The Key Laboratory of Infection and Immunity of Shandong Province, Department of Pharmacology, School of Basic Medical Sciences, Shandong University, Jinan, China

\section{Keywords}

Histone deacetylase - Epigenetic · Podocytes · Proteinuric kidney diseases

\begin{abstract}
Background: Podocytes (highly specialized and terminally differentiated epithelial cells) are integral components of the glomerular filtration barrier that are vulnerable to a variety of injuries and, as a result, they undergo a series of changes ranging from hypertrophy to detachment and apoptosis. Podocyte injury is a major determinant in proteinuric kidney disease and identification of potential therapeutic targets for preventing podocyte injury has clinical importance. Although numerous studies have achieved dramatic advances in the understanding of podocyte biology and its relevance to renal injury, few effective and specific therapies are available. Summary: Epigenetic modifications have been proven to play important roles in the pathogenesis of kidney diseases. Among them, histone deacetylase (HDAC)-mediated epigenetic acetylation in the kidney has attracted much attention, which may play multiple roles in both kidney development and the pathogenesis of kidney disease. Recent studies have demonstrated that HDAC protect against podocyte injury by regulation of inflammation, apoptosis, autophagy, mitochondrial function, and insulin resistance. In
\end{abstract}

this review, we summarize recent advances in the understanding of the functions and regulatory mechanisms of HDAC in podocytes and associated proteinuric kidney diseases. In addition, we provide evidence of the potential therapeutic effects of HDAC inhibitors for proteinuric kidney disease. Key Messages: Pharmacological targeting of HDACmediated epigenetic processes may open new therapeutic avenues for chronic kidney disease.

(c) 2020 The Author(s)

Published by S. Karger AG, Basel

\section{Introduction}

Podocytes are highly specialized and terminally differentiated epithelial cells that are essential for formation and maintenance of the glomerular filtration barrier [1, 2]. Despite compelling evidence identifying the podocyte as the key injury mediator in the pathogenesis of proteinuric kidney diseases such as diabetic nephropathy (DN), focal segmental glomerulosclerosis, membranous nephropathy, and minimal change disease [2], cell-specific therapies are not clinically available, and validated therapeutic targets are scarce. Therefore, identifying the key and universal molecules involved in the different forms of podocytopathies may provide clues to develop new 
Table 1. Size, cellular localization, and activity of HDAC

\begin{tabular}{ccll}
\hline Classification & $\begin{array}{c}\text { Size (amino } \\
\text { acids) }\end{array}$ & Cellular localization & Activity \\
\hline Class I & & & \\
HDAC1 & 482 & Nuclear & \\
HDAC2 & 488 & Nuclear & Deacetylase \\
HDAC3 & 428 & Nuclear/cytoplasmic & Deacetylase \\
HDAC8 & 377 & Nuclear & Deacetylase \\
Class IIa & & & Deacetylase \\
HDAC4 & 1,084 & Nuclear/cytoplasmic & \\
HDAC5 & 1,122 & Nuclear/cytoplasmic & Deacetylase \\
HDAC7 & 952 & Nuclear/cytoplasmic & Deacetylase \\
HDAC9 & 1,011 & Nuclear/cytoplasmic & Deacetylase \\
Class IIb & & Deacetylase \\
HDAC6 & 1,215 & Cytoplasmic & \\
HDAC10 & 669 & Cytoplasmic & Deacetylase \\
Class III & & & Deacetylase \\
Sirt1 & 747 & Nuclear/cytoplasmic & \\
Sirt2 & 389 & Cytoplasmic & Deacetylase \\
Sirt3 & 399 & Nuclear/mitochondrial & Deacetylase \\
Sirt4 & 314 & Mitochondrial & Deacetylase \\
Sirt5 & 310 & Mitochondrial & ADP-ribosyl transfeace \\
Sirt6 & 355 & Nuclear/cytoplasmic & Deacetylase \\
Sirt7 & 400 & Nuclear & Deacetylase/ADP-ribosyl transfeace \\
Class IV & 347 & Nuclear & Deacetylase \\
HDAC11 & & & \\
\hline & & Deacetylase \\
\hline
\end{tabular}

therapeutic strategies for patients with proteinuric kidney diseases. Emerging evidence has shown that histone deacetylase (HDAC)-mediated epigenetic modification plays an important role in the regulation of podocyte function [3]. In this review, we summarize recent advances in the understanding of the functions and regulatory mechanisms of HDAC in podocytes and associated proteinuric kidney diseases. Pharmacological targeting of HDAC-mediated epigenetic processes may open new therapeutic avenues for CKD.

\section{Classification and Cellular Localization of HDAC}

Epigenetics is the study of heritable changes in gene expression that do not involve changes to the underlying DNA sequence - a change in phenotype without a change in genotype. Epigenetic change is a regular and natural occurrence but it can also be influenced by several factors including age, environmental conditions, and disease state. Epigenetic modifications consist of at least 3 systems including DNA methylation, histone modification, and noncoding RNA-associated regulation. Among them, histone or nonhistone protein acetylation mediated by HDAC, which balances the acetylation activities of histone acetyltransferases (HAT) on chromatin remodeling, plays essential roles in the regulation of cellular functions (Table 1). The family of HDAC consists of at least 18 members, which are grouped into 4 classes, including classes I, II, III, and IV [4]. Class I HDAC (HDAC1, 2, 3, and 8), class II HDAC (HDAC4-7, 9, and 10), and class IV (HDAC11) are $\mathrm{Zn}^{2+}$-dependent deacetylases (Fig. 1). Class I HDAC share sequence homology with the yeast Rpd 3 protein and contain a nuclear localization signal but, with the exception of HDAC3, no nuclear export signal. Accordingly, they are mainly located in the nucleus. Class II HDAC are related to the yeast $H d a 1$ gene and further subdivided into classes IIa (HDAC4, 5, 7, and 9) and IIb (HDAC6 and 10). Compared with class I, class II HDAC are larger proteins because they contain additional regulatory domains - nuclear export signals - and as a result they can shuttle between the cytoplasm and the nucleus according to their phosphorylation status. HDAC11 is the sole member of class IV that is localized in the nucleus with a catalytic domain in the N-terminal region. However, it keeps unclear the function of HDAC11 in the 


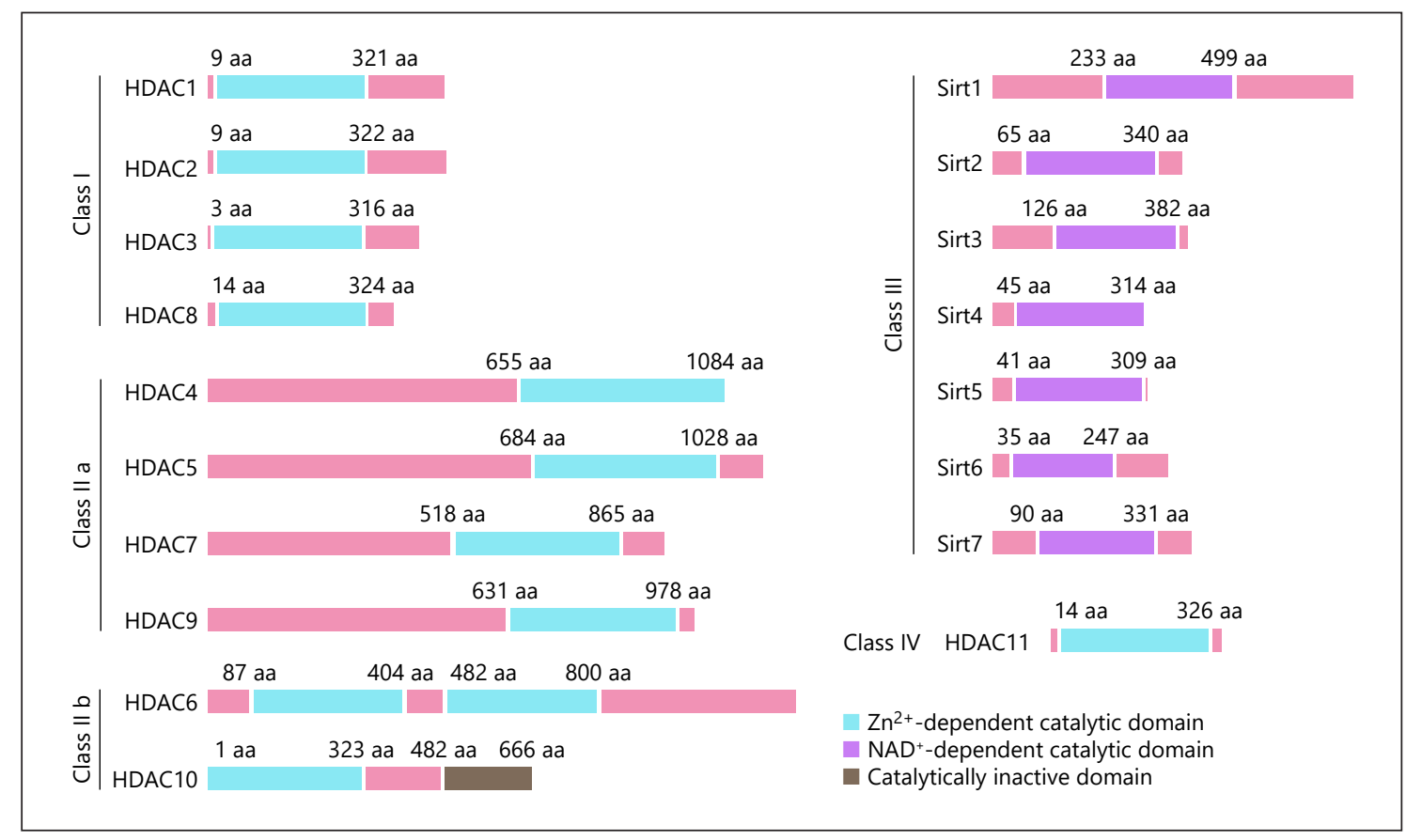

Fig. 1. Structure of HDAC. The family of HDAC consists of at least 18 members, which are grouped into 4 classes, i.e., classes I, II, III, and IV. Class I HDAC (HDAC1, 2, 3, and 8), class II HDAC (HDAC4-7, 9, and 10), and class IV (HDAC11) are $\mathrm{Zn}^{2+}$-dependent deacetylases. The class III HDAC (also known as the Sirtuins) are composed of 7 isoforms, i.e., Sirt1 to Sirt7. aa, amino acids.

kidney. The major biological function of $\mathrm{Zn}^{2+}$-dependent HDAC in epigenetic regulation is thought to be its role as a competitor for HAT through catalyzing of the deacetylation of $\mathrm{N}$-terminal tails of histone proteins, which facilitates condensation of the chromatin structure to prevent transcription factor access to DNA, thus leading to transcriptional repression. In fact, the effect of HDAC in the balance of acetylation and deacetylation is not restricted to histone proteins. More than 50 acetylated nonhistone proteins such as GATA binding protein (GATA), nuclear transcription factor (NF)- $\kappa \mathrm{B}, \mathrm{p} 53$, and hypoxiainducible factor (HIF)- $1 \alpha$, could act as substrates for HDAC in various physiological and pathological processes [5].

Sirtuins (Sirts), also known as class III HDAC, are nicotinamide adenine dinucleotide $\left(\mathrm{NAD}^{+}\right)$-dependent deacetylases that play important roles in transcription regulation, energy metabolism modulation, cell survival, and DNA repair [6]. Currently, the sirtuin family consists of 7 numbers (Sirt1 to Sirt7) with different subcellular localizations and functions. Although Sirt1-7 share conserved core catalytic domains (Fig. 1), the subcellular localization of Sirts has always been an issue of debate. Sirt1,
Sirt6, and Sirt7 are mainly localized in the nucleus, modulating gene expression [7]. However, recent studies have found that Sirtl is also expressed in the cytoplasm in some neuron-like cells of the striatum and in ependymal cells [8]. Although Sirt6 is originally identified as a nuclearlocalizing protein and our previous studies confirmed that Sirt6 is localized in the nucleus of human podocytes, we have also observed that Sirt6 is more or less expressed in the cytoplasm of other renal parenchymal cells, leading us to speculate that renal Sirt6 may shuttle between the nucleus and the cytoplasm, depending on cell types or different environmental stimuli [9]. Sirt3, Sirt4, and Sirt5 are mainly localized in mitochondria, where they regulate the activities of metabolic and energetic enzymes [7]. Compared to the processed short form of Sirt3 that is a wellestablished mitochondrial protein, full-length Sirt 3 is observed in the nucleus in HeLa cells [10]. Sirt2 is considered a cytoplasmic protein. Collectively, Sirts are distributed among multiple compartments of the cell, and their localizations may be dynamic, depending on tissue/cell types or physiologic conditions. Besides their subcellular localization, different members of Sirts exhibit different biological functions (Table 1). Sirt6 has been 
proven to possess deacetylase activity and adenosine diphosphate (ADP)-ribosyl transferase activity [11]. However, Sirt4 is shown to be an ADP-ribosyl transferase without deacetylase activity [12]. Functionally, Sirts promote chromatin silencing and transcriptional repression through deacetylation of histones, such as H4 Lys16 (H4K16), H3 Lys9 (H3K9), H3 Lys14 (H3K14), and H1 Lys26 (H1K26), and then regulate gene transcription [11]. On the other hand, Sirts can also mediate acetylation of nonhistone proteins, such as transcription factors and transcriptional coregulatory proteins.

In this review, we summarize recent advances in the understanding of the functions and regulatory mechanisms of HDAC in podocytes and associated proteinuric kidney diseases.

\section{$\mathrm{Zn}^{2+}$-Dependent HDAC on Regulation of Podocyte Function}

As it is an important organ, kidney development begins in the embryonic stage. In the process, some abnormalities occur and lead to congenital anomalies of the kidney and urinary tract, which account for up to $30 \%$ of end-stage renal disease in children aged $<4$ years [13]. Recent studies have revealed a central role of HDAC in the control of kidney development. These findings provide new insights into the epigenetic mechanisms underlying congenital anomalies of the kidney and urinary tract, implicating the potential of HDAC as therapeutic targets in kidney diseases, such as cystic kidney diseases. Studies have found that the HAT CBP and p300 are necessary for maintenance of renin cell identity and structural integrity of the kidney, indicating that histone acetylation is involved in kidney development [14]. Furthermore, Chen et al. [15] systematically illustrated the expression profile of HDAC and found that class I HDAC1, 2, and 3, and class II HDAC4, 7, and 9 are elevated prenatally but declines upon maturation into the adult kidney. However, HDAC5, 6, and 8 are constitutively expressed [15]. Immunofluorescence results further revealed that HDAC1, 2, and 3 are mainly distributed in undifferentiated metanephric mesenchyme, dichotomizing ureteric branches, and the stroma [15]. Long-term treatment of embryonic kidneys with HDAC inhibitors can damage the ureteric bud branching morphogenesis program and provokes growth arrest and apoptosis [15]. In addition, nephron progenitor cell-specific deletion of $H D A C 1$ and $H D A C 2$ genes in mice has been reported to result in early postnatal lethality due to renal hypodysplasia and loss of nephron progenitor cells [13]. However, studies have also found that inhibition of HDAC expands the renal progenitor cell population [16]. The apparent contradiction in the outcome of HDAC inhibition on cellular proliferation may be intrinsic to differences in pronephros versus metanephros morphogenesis or the specificity of HDAC inhibitors.

More importantly, HDAC have also been implicated in the pathogenesis of kidney disease. Recent studies from our lab have unraveled the expression patterns of HDAC and found that, among $\mathrm{Zn}^{2+}$-dependent HDAC, HDAC2, 4 , and 5 are upregulated in the kidneys of streptozotocin (STZ)-induced diabetic rats and diabetic $d b / d b$ mice and in kidney biopsies from diabetic patients [17]. Podocytes treated with common detrimental factors under diabetic condition such as high glucose, advanced glycation end products, or transforming growth factor- $\beta$ selectively induce HDAC4 expression [17]. We further demonstrated that podocyte injury is associated with suppression of autophagy and exacerbation of inflammation by HDAC4mediated signal transducers and activators of transcription factor 1 (STAT1) signaling [17]. A very recent study also confirmed that podocyte HDAC activity regulates murine and human glomerular diseases [18]. It is found that HDAC1 and HDAC2 activities are increased in mice podocytopathy models and podocyte-associated HDAC1 and $H D A C 2$ genetic ablation improves proteinuria and glomerulosclerosis through regulation of early growth response 1 (EGR1) [18]. Furthermore, when studying the toxic effects of prenatal caffeine exposure (PCE) on podocyte development in male offspring, Zhu et al. [19] found that HDAC7 can reduce Krüppel-like factor 4 (KLF4) expression via downregulation of the $\mathrm{H} 3 \mathrm{~K}$ 9ac level in the KLF4 promoter, resulting in podocyte developmental toxicity induced by PCE in male offspring rats. Of course, HDAC also act as downstream targets in the podocyte signaling network. For example, miR-29a signaling accelerates podocyte apoptosis, proteinuria, and renal fibrosis through promotion of HDAC4-dependent nephrin deacetylation and ubiquitination [20] (Fig. 2a).

\section{Therapeutic Effects of $\mathrm{Zn}^{2+}$-Dependent HDAC Inhibitors in Proteinuric Kidney Diseases}

HDAC inhibitors are chemical compounds that block the activities of HDAC typically by binding to the zinccontaining catalytic domain of HDAC. According to their chemical structure, HDAC inhibitors are divided into 4 classes, including hydroxamic acids, short-chain 
fatty acids, cyclic peptides, and benzamides. Hydroxamic acids combine with the zinc ion at the catalytic site of HDAC and exert nonspecific HDAC inhibition activity affecting all classes of HDAC. Suberoylanilide hydroxamic acid (SAHA; also known as vorinostat) is the first HDAC inhibitor of hydroxamic acids for the treatment of human cutaneous T-cell lymphoma approved by the US Food and Drug Administration (USFDA) [21]. Short chain fatty acids, such as valproic acid (VPA) and sodium butyrate selectively inhibit class I and IIa HDAC and have less potent inhibitory effects compared to hydroxamic acids [21]. The best representative of this class is VPA. Cyclic peptides are one group of $\mathrm{Zn}^{2+}$-dependent HDAC inhibitors with a relatively high selectivity. Among them, romidepsin (depsipeptide, FK-228) was approved by the USFDA for the treatment of cancer in 2009 due to its potency to arrest cell growth [21]. Benzamides are considered a kind of HDAC isoform-selective inhibitor with a long half-life [21].

The protective effect of VPA in podocytes from mice with adriamycin (ADR) nephropathy was firstly assessed. It has been found that VPA decreases the level of proteinuria and maintains the ultrastructure of podocytes [22]. VPA prevents the development of proteinuria and the onset of glomerulosclerosis when it is given before the ADR insult [22]. Moreover, postponing the VPA treatment, until a significant peak in proteinuria is observed, also results in a drop in proteinuria [22]. These data indicate that VPA treatment in an early phase of renal disease can halt and even prevent the development of proteinuria and the progression of kidney damage. In another animal model, VPA or SAHA was demonstrated to mitigate foot process effacement of podocyte and proteinuria in

Fig. 2. a Mechanisms of $\mathrm{Zn}^{2+}$-dependent HDACs in podocyte injury. The activation of HDAC induces podocyte injury by regulation of EGR1. Moreover, upregulation of HDAC4 decreases autophagy to mitigate podocyte injury by upregulating the acetylated level of STAT1. MiR-29a signaling accelerates podocyte apoptosis, proteinuria, and renal fibrosis through promotion of HDAC4-dependent effects, which negatively regulates the expression of miR29a via deacetylation of $\mathrm{H} 3 \mathrm{~K} 9$. In addition, HDAC7 can reduce KLF4 expression by downregulating the H3K9ac level in the KLF4 promoter, resulting in podocyte developmental toxicity induced by PCE in male offspring rats. $\mathbf{b}$ Effects of deacetylation on histone mediated by Sirts in podocytes. Under normal conditions, Sirts could deacetylate the histone and then inhibit gene transcription. However, protein levels of Sirt1 and Sirt6 are downregulated in podocyte injury, and transcription is activated. For example, the decrease in Sirt1 enhances the level of histone acetylation on $\mathrm{H} 3$
Tln $1^{f l f l}$ Pod-rtTA TetO-Cre mice, which constitutes an adult genetic mouse model for proteinuric kidney disease by ablating $T \ln 1$ in podocytes in a conditional doxycycline-inducible manner [18]. Very excitedly, VPA was demonstrated to reduce the mean annual decrease in the estimated glomerular filtration rate in proteinuric patients from a longitudinal analysis of the multicenter Veterans Aging Cohort Study [18]. Furthermore, it has been found that VPA ameliorates podocyte and renal injuries mainly by facilitating the autophagy and inactivation of NF- $\kappa \mathrm{B} /$ inducible nitric oxide synthase (iNOS) signaling in DN [23]. Besides podocytes, VPA also significantly attenuates tubule cell injury and renal fibrosis, as well as proteinuria and glomerular matrix accumulation [24, 25]. Vorinostat, as the first HDAC inhibitor approved by the USFDA, has been reported to reduce tubule cell proliferation, glomerular hypertrophy, and renal enlargement in STZ-diabetic rats [26]. Moreover, vorinostat attenuates albuminuria and reduces glomerular collagen IV deposition and mesangial matrix accumulation in STZdiabetic endothelial nitric oxide synthase (eNOS) knockout mice [27]. Besides VPA and vorinostat, other HDAC inhibitors have also been reported in the treatment of animals with DN. Sodium buthrate lowers plasma glucose and prevents fibrosis and albuminuria in $\mathrm{DN}[28,29]$. Noh et al. [30] demonstrated that trichostatin A and SK7041 decreases matrix production in STZ-diabetic rats. Although an increasing number of studies report the potential clinical benefits of HDAC inhibitors in chronic kidney diseases, none of them has adopted it into clinical trials because of tolerability and isoform-selectivity. Therefore, further studies are needed to continue deciphering the role of individual HDAC in the kidney in dif-

and $\mathrm{H} 4$, leading to a reduction in histone methylation of $\mathrm{H} 3 \mathrm{~K}$, which then promotes Claudin-1 transcription and ultimately causes podocyte injury. In addition, Sirt6 protects against podocyte injury and proteinuria in DN and focal segmental glomerulosclerosis, at least in part through epigenetic regulation of Notch signaling via binding to their promoters and deacetylating $\mathrm{H} 3 \mathrm{~K}$. c Effects of deacetylation on nonhistone mediated by Sirts in podocytes. Sirt1 regulates deacetylation of PGC-1 $1 \alpha$ and cortactin and then improves mitochondrial function and maintains the cytoskeleton. Moreover, Sirt1 could also deacetylate FOXO3, FOXO4, p65 $\mathrm{NF}-\kappa \mathrm{B}$, and STAT3, followed by attenuation of podocyte injury via anti-inflammation, antiapoptosis, reduction of oxidative stress, and inhibition of senescence. In addition, recent studies have demonstrated that colocalization of Sirt3 and KLF15 leads to deacetylation of KLF15, with decreased expression of fibrosis factors in podocytes with AngII treatment.

(For figure see next page.) 


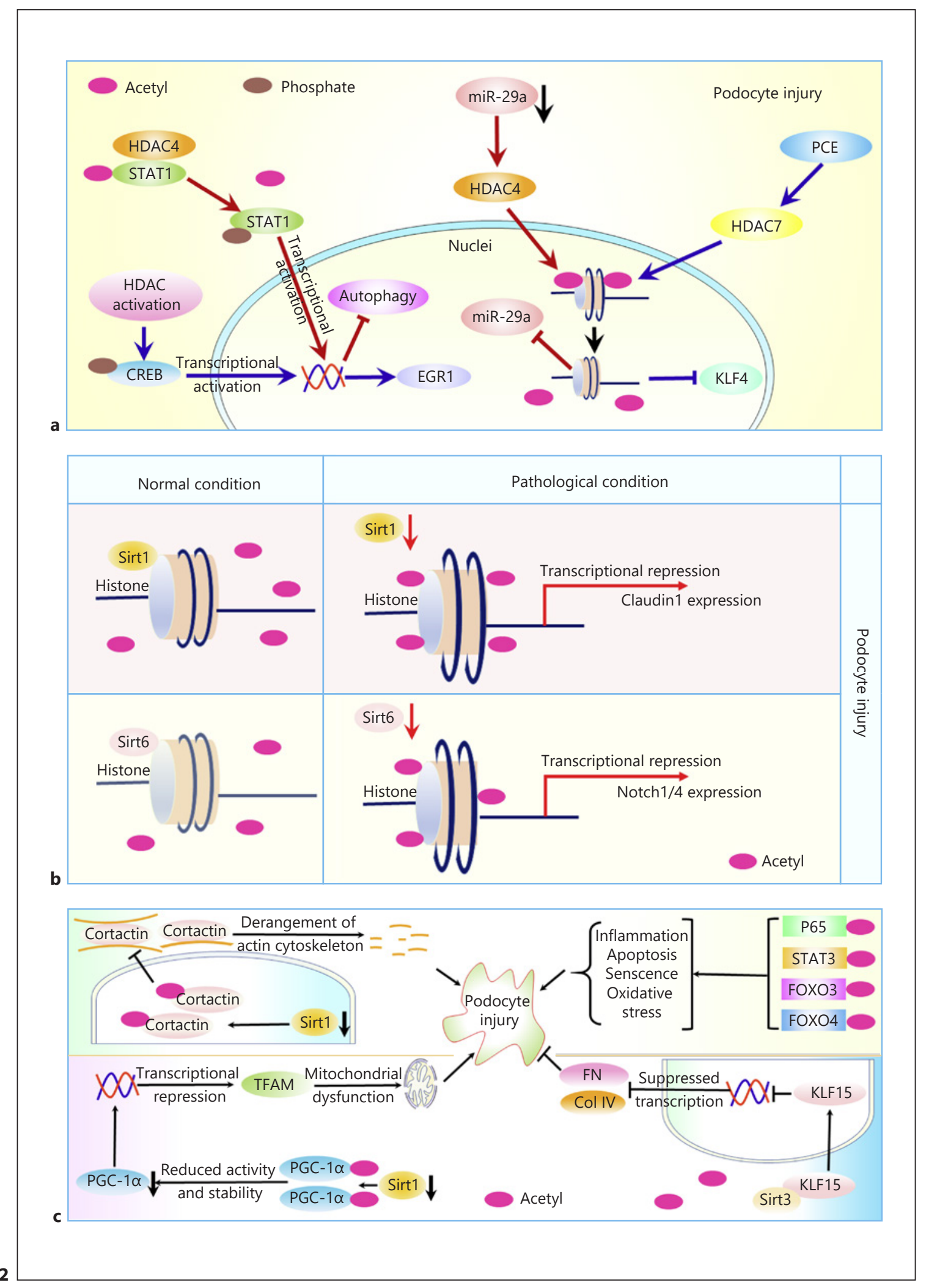


Table 2. Null phenotype of different members of the Sirts

\begin{tabular}{ll}
\hline Knockout mice & Phenotype \\
\hline Sirt1 $^{-/-}$ & Developmental defects, lethal in some backgrounds \\
\hline Sirt3 $^{-/-}$ & $\begin{array}{l}\text { No obvious phenotype, though biochemical phenotypes, such as AceCS2 activity and } \\
\text { ATP levels, have been reported }\end{array}$ \\
\hline Sirt4 $^{-/-}$ & Increased mitochondrial GDP activity (normal activity exhibited in mice) \\
\hline Sirt5 $^{-/-}$ & Defect in the urea cycle \\
\hline Sirt6 $^{-/-}$ & Severe hypoglycemia, premature aging \\
\hline Sirt7 & Smaller size, reduced lifespan, heart defects \\
\hline
\end{tabular}

AceCS2, acetyl-CoA synthetase 2; ATP, adenosine triphosphate; GDP, guanosine diphosphate.

ferent physiological and pathological situations and develop isoform-specific HDAC inhibitors for optimized therapy in the near future.

\section{NAD $^{+}$-Dependent Deacetylases on the Regulation of Podocyte Function}

Aging is a universal process that affects multiple organs, and the kidney is one of the most vulnerable organs. Podocytes are integral components of the renal glomerular filtration barrier and have a limited ability to repair and regenerate themselves [2]; therefore, podocyte aging or injury plays a central role in renal aging [31,32]. Sirts have been proven to be closely associated with a number of age-related diseases, such as neurodegenerative disease [33], metabolic disease, and kidney disease [34]. Because mice homozygous for Sirt1 deletion fail to thrive [35] (Table 2), analysis of the functional significance of Sirt1 in podocytes is often aided by genetically engineered mice with inducible and reversible Sirt1 knockdown in widespread, heterozygous Sirt1-null mice or podocyte-specific Sirt1 deletion mice [36]. Heterozygous Sirt1-null mice $\left(\right.$ Sirt $\left.^{+/-}\right)$or podocyte-conditional Sirt1 knockout mice cannot develop any abnormality in renal histological characteristics or urinary protein excretion in youth [36]. However, the reduction of podocyte Sirt1 leads to aggravated aging-induced glomerulosclerosis and albuminuria [37]. In addition, the urinary level of 8-hydroxy-2'-deoxyguanosine, a marker for oxidative stress, is markedly increased in aged podocyte conditional knockout Sirt1 mice compared to controls [37]. Although podocyte-specific markers decrease in aged mice compared to young controls, the decrease is further exacerbated in aged podocyte conditional knockout Sirt1 mice [37]. Furthermore, the expression of cellular senescence markers is significantly higher in isolated glomeruli from podocyte conditional knockout Sirt1 mice, indicating that Sirt1 may be a potential therapeutic target to treat patients with aging-related kidney disease [37]. Interestingly, recent studies have also found that the density of tight pores is higher in proximal tubule (PT) Sirt1-specific knockout mice than in wild-type mice, as is the increase in urinary albumin excretion, indicating that endogenous PT Sirt1 may have a protective effect on podocytes under normal conditions [38]. Moreover, Liu et al. [9] also found that podocyte-specific deletion of Sirt6 mice can cause development a slight albuminuria and podocyte injury at 12 month age, though no significant difference was observed in podocyte-specific deletion of Sirt6 mice compared to their control littermates when followed up to 9 months after birth. Consistently, global Sirt6 deletion in mice induces podocyte injury as evidenced by decreases in slit diaphragm proteins and foot process effacement, eventually leading to proteinuria [39]. These results suggest that Sirt1 and Sirt6 may be potential therapeutic targets for the treatment of patients with aging-related kidney disease. Nevertheless, the function of other members of Sirts in podocyte physiology remains unclear. Therefore, it is necessary to further clarify their functions in podocytes.

Under pathophysiological conditions, a reduction of Sirts contributes to podocyte injuries. In fact, some studies have reported that the podocyte-protective effect of Sirts may originate from renal tubules. It has been found that the level of Sirt1 is decreased in PT before glomeruli in diabetic mice [38]. PT-specific knockout of Sirt1 exacerbates podocyte injury and proteinuria in $\mathrm{DN}$, while $\mathrm{PT}$ specific transgenic of Sirt1 can significantly ameliorate 
renal injury as indicated by reduced albuminuria and podocyte injury as evidenced by foot process broadening and effacement [38]. Mechanistically, the decrease in Sirtl increases the level of histone acetylation on $\mathrm{H} 3$ and $\mathrm{H} 4$, leading to a reduction in the histone methylation of H3K9, which promotes claudin-1 transcription and ultimately results in podocyte injury [38] (Fig. 2b). These results reveal a novel mechanism whereby tubular epithelial cells affect the podocyte epigenome though Sirt1-mediated epigenetic regulation in $\mathrm{DN}$, which has been named as "PT-podocyte communication" [40]. In DN, Sirtl expression is also reduced in podocytes from the kidneys of $d b / d b$ mice [41]. Gene deficiency of Sirt1 in podocytes aggravates proteinuria and kidney injury by promoting acetylation of $\mathrm{p} 65$ and activators of transcription factor 3 (STAT3) in $d b / d b$ mice [41] (Fig. 2c). Overexpression of Sirt1 or treatment of BF175, a potent and selective Sirt1 agonist, could markedly ameliorate glomerular injury in diabetic OVE26 mice [42]. In addition, Sirt1 knockdown enhances the width of podocyte foot processes and the secretion of proteinuria in ADR-induced nephropathy [36]. The protective effect of Sirt1 on podocytes has also been proven in glomerular disease induced by nephrotoxic serum [43]. Podocyte-specific knockout of Sirt1 causes the accumulation of acetylated cortactin in the nucleus and less deacetylation of cortactin in the cytoplasm, thereby aggravating podocyte injury [43] (Fig. 2c). Furthermore, more and more studies have demonstrated that Sirt1 possesses variously protective effects, i.e., anti-inflammation, inhibition of apoptosis, improvement of insulin resistance, increase in autophagy, and maintenance of mitochondrial function [44-48]. These studies suggest that Sirt1 may be a universal molecule involved in the different forms of podocytopathies.

Additionally, Sirt3 is downregulated in the kidneys of mice subjected to chronic angiotensin II (AngII) infusion [49]. Sirt 3 knockout aggravates podocyte injury by directly deacetylating KLF15, resulting in more serious hypertensive nephropathy [49] (Fig. 2c). Sirt3 has also been demonstrated to protect against podocyte injury as the target of the membrane $G$ protein-coupled receptor 5 (TGR5) in kidney injuries caused by obesity and diabetes mellitus [50]. In addition, Shiraishi et al. [51] provided direct evidence that nicorandil may synergize with enalapril to alleviate chronic kidney injuries via regulation Sirt3. Our studies have highlighted the role of Sirt6 in podocytes, which has pleiotropic protective actions including anti-inflammatory and antiapoptotic effects, maintenance of the actin cytoskeleton, and promotion of autophagy [9]. Liu et al. [9] found that Sirt6 was down- regulated in renal biopsies from patients with podocytopathies, such as focal segmental glomerulosclerosis, membranous nephropathy, DN and IgA nephropathy, and correlated with glomerular filtration rate. Podocytespecific deletion of Sirt6 exacerbated podocyte injury and proteinuria in 2 independent mouse models including DN and ADR-induced nephropathy [9]. Mechanistically, Sirt6 negatively regulates Notch signaling by suppression of the transcription of Notch 1 and Notch4 genes [9] (Fig. 2b). Recent studies have also found that Sirt6 protects mitochondria of podocytes and exerts antiapoptotic effects via activation of the AMP-activated protein kinase (AMPK) pathway [52]. Collectively, pharmacological targeting of Sirt6-mediated Notch signaling pathways at multiple levels may provide a novel approach for the treatment of proteinuric kidney disease.

\section{Effects of Sirt Modulators on Podocyte Function}

The potential involvement of Sirts in the pathogenesis of kidney diseases gives rise to the development of smallmolecule modulators, including inhibitors and activators. Although several promising inhibitors have been developed, the clinical potential of Sirt inhibitors is mostly unknown because of their potency, isotype selectivity, or suitable physicochemical properties [53]. Selisistat is the only Sirt1 inhibitor that has reached clinical trials for the treatment of Huntington's disease [54]. However, studies of Sirt inhibitors in the kidney are still rare due to the potential protective effects of Sirts in proteinuric kidney disease. With more efforts undertaken to develop smallmolecule activators of Sirt, several compounds such as SRT1720, SRT2183, and SRT3025 have been demonstrated to protect against kidney injury, especially in acute kidney injury [55]. In addition, Resveratrol, an activator of Sirt1, has been shown to attenuate aldosterone-induced mitochondrial dysfunction and podocyte injury via maintenance of peroxisome proliferator-activated receptor- $\gamma$ coactivator $1-\alpha$ (PGC-1 $\alpha)$ expression [44] (Fig. 2c). Resveratrol can also significantly repress highglucose-induced vascular endothelial growth factor (VEGF) and VEGF receptor-2 (Flk-1) expressions in cultured mouse podocytes, modulating angiogenesis in DN [56]. Moreover, other potent Sirt1 agonists such as grape seed procyanidin B2 [57, 58], metformin [59], puerarin [60], and catalpol [61] have also been studied in podocytes. Our very recent studies explore a novel role of progranulin (PGRN) which is a secreted glycoprotein with pleiotropic functions including embryogenesis, inflam- 
mation, wound repair, and lysosome function [62], on the regulation of Sirtl and found that PGRN can maintain mitochondrial homeostasis via PGRN-Sirt1-PGC-1 $\alpha /$ forkhead box protein O 1 (FOXO1) signaling-mediated mitochondrial biogenesis and mitophagy in podocytes [48]. Although these results indicate that the activation of Sirts could be beneficial for patients with kidney disease, initial clinical studies with Sirts activators are inconsistent. Therefore, more extensive studies are required to understand the function of individual Sirts and its therapeutic potential.

\section{Summary and Outlook}

In this review, we summarize the current findings of HDAC in the kidney and outline important molecular mechanisms of HDAC on the regulation of podocyte functions. We also provide evidence showing the therapeutic effects of HDAC inhibitors or Sirt activators for proteinuric kidney disease. It should be noted that although Sirts and other HDAC are considered HDAC, they seem to exert opposite effects on podocytes for several possible reasons. First, Sirts and $\mathrm{Zn}^{2+}$-dependent HDAC are mechanistically and structurally distinct. Sirts show no sequence homology to the $\mathrm{Zn}^{2+}$-dependent HDAC. Sirts transfer an acetyl group from lysine to $\mathrm{NAD}^{+}$, creating $\mathrm{O}$-acetyl ADP-ribose and nicotinamide as the product [53]. However, $\mathrm{Zn}^{2+}$-dependent HDAC cleave the amide bond of $\mathrm{N} \varepsilon$-acetylated lysine residues via a charge-relay system, which acts in a $\mathrm{Zn}^{2+}$-dependent catalysis mechanism and could be inhibited by $\mathrm{Zn}^{2+}$-che- lating agents [53]. In addition, some Sirts such as Sirt4 and Sirt6 perform the function of mono-ADP-ribosyltransferase as well. Second, the substrates of Sirts and $\mathrm{Zn}^{2+}$-dependent HDAC are largely different. Different targets regulate different signaling pathways, eventually inducing different effects in podocytes. Therefore, further studies are needed to continue deciphering the role of individual HDAC in different physiological and pathological situations, although studies have made great progress in the recognition of HDAC. Collectively, pharmacological targeting of HDAC may be an innovative therapeutic strategy for treating patients with kidney disease by epigenetic modifications.

\section{Disclosure Statement}

All the authors declare no competing interests.

\section{Funding Sources}

This study was supported by the National Science Fund for Distinguished Young Scholars (F.Y.; 81525005), the National Nature Science Foundation of China $(81800645,91642204$, and 91949202), the Shandong Provincial Natural Science Foundation (ZR2019BH030), and the China Postdoctoral Science Foundation (2018M640634).

\section{Author Contributions}

M.L., Y.Z., and P.Z. wrote this paper; M.L. and Z.Q. drew the figures; and F.Y. designed and finalized this work.

\section{References}

1 Mathieson PW. The podocyte as a target for therapies-new and old. Nat Rev Nephrol. 2011 Nov;8(1):52-6.

2 Zhou L, Liu Y. Wnt/ $\beta$-catenin signalling and podocyte dysfunction in proteinuric kidney disease. Nat Rev Nephrol. 2015 Sep;11(9): $535-45$

3 Wei Q, Dong Z. HDAC4 blocks autophagy to trigger podocyte injury: non-epigenetic action in diabetic nephropathy. Kidney Int. 2014 Oct;86(4):666-8.

4 Bolden JE, Peart MJ, Johnstone RW. Anticancer activities of histone deacetylase inhibitors. Nat Rev Drug Discov. 2006 Sep;5(9):769-84.

5 Glozak MA, Sengupta N, Zhang X, Seto E. Acetylation and deacetylation of non-histone proteins. Gene. 2005 Dec;363:15-23.
6 Hao CM, Haase VH. Sirtuins and their relevance to the kidney. J Am Soc Nephrol. 2010 Oct;21(10):1620-7.

7 Michishita E, Park JY, Burneskis JM, Barrett JC, Horikawa I. Evolutionarily conserved and nonconserved cellular localizations and functions of human SIRT proteins. Mol Biol Cell. 2005 Oct; 16(10):4623-35.

8 Tanno M, Sakamoto J, Miura T, Shimamoto K, Horio Y. Nucleocytoplasmic shuttling of the NAD+-dependent histone deacetylase SIRT1. J Biol Chem. 2007 Mar;282(9):682332.

9 Liu M, Liang K, Zhen J, Zhou M, Wang X, Wang Z, et al. Sirt6 deficiency exacerbates podocyte injury and proteinuria through targeting Notch signaling. Nat Commun. 2017 Sep;8(1):413.
10 Iwahara $T$, Bonasio R, Narendra V, Reinberg D. SIRT3 functions in the nucleus in the control of stress-related gene expression. $\mathrm{Mol}$ Cell Biol. 2012 Dec;32(24):5022-34.

11 Kitada M, Kume S, Takeda-Watanabe A, Kanasaki K, Koya D. Sirtuins and renal diseases: relationship with aging and diabetic nephropathy. Clin Sci (Lond). 2013 Feb;124(3): 153-64.

12 Guarente L. Franklin H. Epstein Lecture: Sirtuins, aging, and medicine. N Engl J Med. 2011 Jun;364(23):2235-44.

13 Liu H, Chen S, Yao X, Li Y, Chen CH, Liu J, et al. Histone deacetylases 1 and 2 regulate the transcriptional programs of nephron progenitors and renal vesicles. Development. 2018 May;145(10):dev153619. 
14 Gomez RA, Pentz ES, Jin X, Cordaillat M, Sequeira Lopez ML. CBP and p300 are essential for renin cell identity and morphological integrity of the kidney. Am J Physiol Heart Circ Physiol. 2009 May;296(5):H1255-62.

15 Chen S, Bellew C, Yao X, Stefkova J, Dipp S, Saifudeen Z, et al. Histone deacetylase (HDAC) activity is critical for embryonic kidney gene expression, growth, and differentiation. J Biol Chem. 2011 Sep;286(37):3277589.

16 de Groh ED, Swanhart LM, Cosentino CC, Jackson RL, Dai W, Kitchens CA, et al. Inhibition of histone deacetylase expands the renal progenitor cell population. J Am Soc Nephrol. 2010 May;21(5):794-802.

17 Wang X, Liu J, Zhen J, Zhang C, Wan Q, Liu $\mathrm{G}$, et al. Histone deacetylase 4 selectively contributes to podocyte injury in diabetic nephropathy. Kidney Int. 2014 Oct;86(4):71225.

18 Inoue K, Gan G, Ciarleglio M, Zhang Y, Tian $\mathrm{X}$, Pedigo CE, et al. Podocyte histone deacetylase activity regulates murine and human glomerular diseases. J Clin Invest. 2019 Mar; 129(3):1295-313.

19 Zhu Y, Chen H, Zhao X, Li B, He H, Cheng H, et al. Decreased H3K9ac level of KLF4 mediates podocyte developmental toxicity induced by prenatal caffeine exposure in male offspring rats. Toxicol Lett. 2019 Oct;314:63-74.

20 Lin CL, Lee PH, Hsu YC, Lei CC, Ko JY, Chuang PC, et al. MicroRNA-29a promotion of nephrin acetylation ameliorates hyperglycemia-induced podocyte dysfunction. J Am Soc Nephrol. 2014 Aug;25(8):1698-709.

21 Hadden MJ, Advani A. Histone Deacetylase Inhibitors and Diabetic Kidney Disease. Int J Mol Sci. 2018 Sep;19(9):E2630.

22 Van Beneden K, Geers C, Pauwels M, Mannaerts I, Verbeelen D, van Grunsven LA, et al. Valproic acid attenuates proteinuria and kidney injury. J Am Soc Nephrol. 2011 Oct; 22(10):1863-75.

23 Khan S, Jena G, Tikoo K, Kumar V. Valproate attenuates the proteinuria, podocyte and renal injury by facilitating autophagy and inactivation of NF- $\kappa \mathrm{B} / \mathrm{iNOS}$ signaling in diabetic rat. Biochimie. 2015 Mar;110:1-16.

24 Khan S, Jena G, Tikoo K. Sodium valproate ameliorates diabetes-induced fibrosis and renal damage by the inhibition of histone deacetylases in diabetic rat. Exp Mol Pathol. 2015 Apr;98(2):230-9.

25 Sun XY, Qin HJ, Zhang Z, Xu Y, Yang XC, Zhao DM, et al. Valproate attenuates diabetic nephropathy through inhibition of endoplasmic reticulum stress-induced apoptosis. Mol Med Rep. 2016 Jan;13(1):661-8.

26 Gilbert RE, Huang Q, Thai K, Advani SL, Lee $\mathrm{K}$, Yuen DA, et al. Histone deacetylase inhibition attenuates diabetes-associated kidney growth: potential role for epigenetic modification of the epidermal growth factor receptor. Kidney Int. 2011 Jun;79(12):1312-21.
27 Advani A, Huang Q, Thai K, Advani SL, White KE, Kelly DJ, et al. Long-term administration of the histone deacetylase inhibitor vorinostat attenuates renal injury in experimental diabetes through an endothelial nitric oxide synthase-dependent mechanism. Am J Pathol. 2011 May;178(5):2205-14.

28 Khan S, Jena G. Sodium butyrate, a HDAC inhibitor ameliorates eNOS, iNOS and TGF$\beta 1$-induced fibrogenesis, apoptosis and DNA damage in the kidney of juvenile diabetic rats. Food Chem Toxicol. 2014 Nov;73:127-39.

29 Dong W, Jia Y, Liu X, Zhang H, Li T, Huang $\mathrm{W}$, et al. Sodium butyrate activates NRF2 to ameliorate diabetic nephropathy possibly via inhibition of HDAC. J Endocrinol. 2017 Jan; 232(1):71-83

30 Noh H, Oh EY, Seo JY, Yu MR, Kim YO, Ha $\mathrm{H}$, et al. Histone deacetylase- 2 is a key regulator of diabetes- and transforming growth factor-betal-induced renal injury. Am J Physiol Renal Physiol. 2009 Sep;297(3):F729-39.

31 Hodgin JB, Bitzer M, Wickman L, Afshinnia F, Wang SQ, O'Connor C, et al. Glomerular Aging and Focal Global Glomerulosclerosis: A Podometric Perspective. J Am Soc Nephrol. 2015 Dec;26(12):3162-78.

32 Schmitt R, Melk A. Molecular mechanisms of renal aging. Kidney Int. 2017 Sep;92(3):56979.

33 Ajami M, Pazoki-Toroudi H, Amani H, Nabavi SF, Braidy N, Vacca RA, et al. Therapeutic role of sirtuins in neurodegenerative disease and their modulation by polyphenols. Neurosci Biobehav Rev. 2017 Feb;73:39-47.

34 Guan Y, Wang SR, Huang XZ, Xie QH, Xu YY, Shang D, et al. Nicotinamide Mononucleotide, an NAD+ Precursor, Rescues Age-Associated Susceptibility to AKI in a Sirtuin 1-Dependent Manner. J Am Soc Nephrol. 2017 Aug;28(8):2337-52.

35 Finkel T, Deng CX, Mostoslavsky R. Recent progress in the biology and physiology of sirtuins. Nature. 2009 Jul;460(7255):587-91.

36 Chuang PY, Xu J, Dai Y, Jia F, Mallipattu SK, Yacoub $\mathrm{R}$, et al. In vivo RNA interference models of inducible and reversible Sirt1 knockdown in kidney cells. Am J Pathol. 2014 Jul;184(7):1940-56.

37 Chuang PY, Cai W, Li X, Fang L, Xu J, Yacoub $\mathrm{R}$, et al. Reduction in podocyte SIRT1 accelerates kidney injury in aging mice. Am J Physiol Renal Physiol. 2017 Sep;313(3):F621-8.

38 Hasegawa K, Wakino S, Simic P, Sakamaki Y, Minakuchi H, Fujimura K, et al. Renal tubular Sirt1 attenuates diabetic albuminuria by epigenetically suppressing Claudin-1 overexpression in podocytes. Nat Med. 2013 Nov; 19(11):1496-504

39 Huang W, Liu H, Zhu S, Woodson M, Liu R, Tilton RG, et al. Sirt6 deficiency results in progression of glomerular injury in the kidney. Aging (Albany NY). 2017 Mar;9(3): 1069-83.
40 Hasegawa K, Wakino S, Sakamaki Y, Murao$\mathrm{ka} \mathrm{H}$, Umino H, Minakuchi H, et al. Communication from Tubular Epithelial Cells to Podocytes through Sirt1 and Nicotinic Acid Metabolism. Curr Hypertens Rev. 2016;12(2): 95-104.

41 Liu R, Zhong Y, Li X, Chen H, Jim B, Zhou $\mathrm{MM}$, et al. Role of transcription factor acetylation in diabetic kidney disease. Diabetes. 2014 Jul;63(7):2440-53.

42 Hong Q, Zhang L, Das B, Li Z, Liu B, Cai G, et al. Increased podocyte Sirtuin-1 function attenuates diabetic kidney injury. Kidney Int. 2018 Jun;93(6):1330-43.

43 Motonishi S, Nangaku M, Wada T, Ishimoto Y, Ohse T, Matsusaka T, et al. Sirtuin1 Maintains Actin Cytoskeleton by Deacetylation of Cortactin in Injured Podocytes. J Am Soc Nephrol. 2015 Aug;26(8):1939-59.

44 Yuan Y, Huang S, Wang W, Wang Y, Zhang $\mathrm{P}$, Zhu C, et al. Activation of peroxisome proliferator-activated receptor- $\gamma$ coactivator $1 \alpha$ ameliorates mitochondrial dysfunction and protects podocytes from aldosterone-induced injury. Kidney Int. 2012 Oct;82(7):771-89.

45 Rogacka D, Piwkowska A, Audzeyenka I, Angielski S, Jankowski M. SIRT1-AMPK crosstalk is involved in high glucose-dependent impairment of insulin responsiveness in primary rat podocytes. Exp Cell Res. 2016 Dec; 349(2):328-38

46 Shi JX, Huang Q. Glucagon-like peptide-1 protects mouse podocytes against high glucose-induced apoptosis, and suppresses reactive oxygen species production and proinflammatory cytokine secretion, through sirtuin 1 activation in vitro. Mol Med Rep. 2018 Aug;18(2):1789-97.

47 Zhang T, Chi Y, Ren Y, Du C, Shi Y, Li Y. Resveratrol Reduces Oxidative Stress and Apoptosis in Podocytes via Sir2-Related Enzymes, Sirtuins1 (SIRT1)/Peroxisome ProliferatorActivated Receptor $\gamma$ Co-Activator $1 \alpha$ (PGC1a) Axis. Med Sci Monit. 2019 Feb;25:122031.

48 Zhou D, Zhou M, Wang Z, Fu Y, Jia M, Wang $\mathrm{X}$, et al. PGRN acts as a novel regulator of mitochondrial homeostasis by facilitating mitophagy and mitochondrial biogenesis to prevent podocyte injury in diabetic nephropathy. Cell Death Dis. 2019 Jul;10(7):524.

49 Li N, Zhang J, Yan X, Zhang C, Liu H, Shan $\mathrm{X}$, et al. SIRT3-KLF15 signaling ameliorates kidney injury induced by hypertension. Oncotarget. 2017 Jun;8(24):39592-604.

50 Wang XX, Edelstein MH, Gafter U, Qiu L, Luo Y, Dobrinskikh E, et al. G Protein-Coupled Bile Acid Receptor TGR5 Activation Inhibits Kidney Disease in Obesity and Diabetes. J Am Soc Nephrol. 2016 May;27(5):136278.

51 Shiraishi T, Tamura Y, Taniguchi K, Higaki M, Ueda S, Shima T, et al. Combination of ACE inhibitor with nicorandil provides further protection in chronic kidney disease. Am J Physiol Renal Physiol. 2014 Dec; 307(12):F1313-22. 
52 Fan Y, Yang Q, Yang Y, Gao Z, Ma Y, Zhang L, et al. Sirt6 Suppresses High Glucose-Induced Mitochondrial Dysfunction and Apoptosis in Podocytes through AMPK Activation. Int J Biol Sci. 2019 Jan;15(3):701-13.

53 Schiedel M, Robaa D, Rumpf T, Sippl W, Jung M. The Current State of NAD+-Dependent Histone Deacetylases (Sirtuins) as Novel Therapeutic Targets. Med Res Rev. 2018 Jan; 38(1):147-200.

54 Süssmuth SD, Haider S, Landwehrmeyer GB, Farmer R, Frost C, Tripepi G, et al.; PADDINGTON Consortium. An exploratory double-blind, randomized clinical trial with selisistat, a SirT1 inhibitor, in patients with Huntington's disease. Br J Clin Pharmacol. 2015 Mar;79(3):465-76.

55 Morigi M, Perico L, Benigni A. Sirtuins in Renal Health and Disease. J Am Soc Nephrol. 2018 Jul;29(7):1799-809.
56 Wen D, Huang X, Zhang M, Zhang L, Chen J, $\mathrm{Gu} Y$, et al. Resveratrol attenuates diabetic nephropathy via modulating angiogenesis. PLoS One. 2013 Dec;8(12):e82336.

57 Bao L, Cai X, Dai X, Ding Y, Jiang Y, Li Y, et al. Grape seed proanthocyanidin extracts ameliorate podocyte injury by activating peroxisome proliferator-activated receptor- $\gamma \mathrm{co}-$ activator $1 \alpha$ in low-dose streptozotocin-and high-carbohydrate/high-fat diet-induced diabetic rats. Food Funct. 2014 Aug;5(8):187280.

58 Cai X, Bao L, Ren J, Li Y, Zhang Z. Grape seed procyanidin $\mathrm{B} 2$ protects podocytes from high glucose-induced mitochondrial dysfunction and apoptosis via the AMPK-SIRT1-PGC-1a axis in vitro. Food Funct. 2016 Feb;7(2):80515.
59 Rogacka D, Audzeyenka I, Rychłowski M, Rachubik P, Szrejder M, Angielski S, et al. Metformin overcomes high glucose-induced insulin resistance of podocytes by pleiotropic effects on SIRT1 and AMPK. Biochim Biophys Acta Mol Basis Dis. 2018 Jan;1864(1): $115-25$

60 Li XL, Cai WJ, Lee K, Liu BH, Deng YY, Chen $\mathrm{YP}$, et al. Puerarin attenuates diabetic kidney injury through the suppression of NOX4 expression in podocytes. Sci Rep. 2017 Nov 6; 7(1):14603.

61 Zhang J, Bi R, Meng Q, Wang C, Huo X, Liu $Z$, et al. Catalpol alleviates adriamycin-induced nephropathy by activating the SIRT1 signalling pathway in vivo and in vitro. $\mathrm{Br} \mathrm{J}$ Pharmacol. 2019 Dec;176(23):4558-73.

$62 \mathrm{Fu}$ Y, Sun Y, Zhou M, Wang X, Wang Z, Wei $X$, et al. Therapeutic Potential of Progranulin in Hyperhomocysteinemia-Induced Cardiorenal Dysfunction. Hypertension. 2017 Feb; 69(2):259-66. 